\title{
Triangulations with Very Few Geometric Bistellar Neighbors*
}

\author{
F. Santos \\ Departamento de Matemáticas, Estadística y Computación, \\ Universidad de Cantabria, E-39071 Santander, Spain \\ santos@matesco.unican.es \\ http://matsun1.unican.es/ $/$ santos
}

\begin{abstract}
We are interested in a notion of elementary change between triangulations of a point configuration, the so-called bistellar flips, introduced by Gel'fand, Kapranov, and Zelevinski. We construct sequences of triangulations of point configurations in dimension 3 with $n^{2}+2 n+2$ vertices and only $4 n-3$ geometric bistellar flips (for every even integer $n$ ), and of point configurations in dimension 4 with arbitrarily many vertices and a bounded number of flips. This drastically improves previous examples and seems to be evidence against the conjecture that any two triangulations of a point configuration can be joined by a sequence of flips.
\end{abstract}

\section{Introduction}

Given a finite point configuration $\mathcal{A}$ in the Euclidean space $\mathbb{R}^{d}$ of dimension $d$ we call triangulations of $\mathcal{A}$ all the geometrically realized simplicial complexes which cover the convex hull of $\mathcal{A}$ and which have their sets of vertices contained in $\mathcal{A}$. In this paper we are interested in a notion of vicinity or elementary change between triangulations of a given point configuration $\mathcal{A}$ known as a geometric bistellar flip (or flip, for short).

This notion naturally arises in the theory of secondary and fiber polytopes [2], [9, Chapter 7], [15], [21, Lecture 10]. In this theory, given a point configuration of dimension $d$ with $n$ points, the regular [2], [12], [21] (also called coherent [9] or sometimes convex) triangulations of $\mathcal{A}$ are defined as those which coincide with an orthogonal projection of the lower facets of a simplicial polytope of dimension $d+1$. The secondary polytope of $\mathcal{A}$, introduced by Gel'fand et al. (see [9]) is an $(n-d-1)$-polytope whose vertices are in bijection with regular triangulations of $\mathcal{A}$ and whose edges are in correspondence with

\footnotetext{
* This research was partially supported by Grant PB97-0358 of the Spanish Dirección General de Enseñanza Superior e Investigación Científica.
} 
bistellar flips between regular triangulations (here $n$ is the cardinality of $\mathcal{A}$; the number $n-d-1$ will be called the corank of $\mathcal{A}$ ).

These properties of the secondary polytope imply that the collection of regular triangulations of $\mathcal{A}$ is connected under bistellar flips and that any regular triangulation has at least $n-d-1$ flips. Nonregular triangulations with less than $n-d-1$ flips have been constructed by de Loera et al. in [6] and [8]. We call such triangulations fip-deficient. For example, a construction from [8] gives, for each integer $n$, triangulations in $\mathbb{R}^{3}$ with $5 n$ points and only $3 n-2$ bistellar flips. In the following sections we drastically improve these results by showing constructions of:

- For any positive even integer $n$, a triangulation of a point configuration in $\mathbb{R}^{3}$ with $n^{2}+2 n+2$ vertices and $4 n-3$ flips (Theorem 4 ).

- Triangulations of point configurations in $\mathbb{R}^{4}$ with an arbitrarily large number of vertices and a bounded number of flips (Theorem 10). The number of flips obtained is easier to analyze in a vector configuration instead of a point configuration setting. For any positive integer $n$ we construct a simplicial fan covering the vector space $\mathbb{R}^{5}$, with $15 n+2$ rank-1 cones (vertices) and only nine bistellar flips (Theorem 12). For a point configuration in $\mathbb{R}^{4}$ we can achieve a number of 21 flips, and an arbitrarily large number of vertices (Remark 13).

We are interested in triangulations with few flips as an approach to the question of whether any two triangulations of a point configuration can be connected by a sequence of geometric bistellar flips. The answer is known to be positive in the cases of dimension at most $2(d \leq 2)$ or corank at most $3(n \leq d+4)$. In these cases it is also known that no flip-deficient triangulations exist. Different proofs of connectivity in the case $d \leq 2$ exist, the oldest written one is probably in [11]. That no flip-deficiency exists in this case is proved in [8]. Connectivity and no-flip-deficiency in the case $n \leq d+3$ follow from the fact, proved by Lee [12], that in this case all the triangulations are regular. The case $n=d+4$ is a recent result of Azaola and Santos [1], whose proof is based on the notion of virtual chamber introduced in [7].

Another interesting case is that of cyclic polytopes. Rambau [15] has proved that the set of triangulations is connected by flips when $\mathcal{A}$ is the collection of vertices of a cyclic polytope $C(n, d)$. On the other hand, there are triangulations of $C(11,5)$ with only four flips, instead of five [16].

No negative example to the connectivity question is known, and the question itself is a weak version of the so-called Baues problem for triangulations, posed by Billera et al. [4] (see also [14], [17], and [18]). The Baues problem asks whether the refinement poset of all subdivisions of a point configuration $\mathcal{A}$ with $n$ points in dimension $d$ is homotopy equivalent to an $(n-d-2)$-sphere. The question has connections to oriented matroid theory [5, Section 9.6], zonotopal tilings (via the Bohne-Dress theorem [5], [21]), and combinatorial differential geometry, as introduced by MacPherson [13].

The fact that we can construct triangulations with very few geometric bistellar flips seems to be evidence in favor of the existence of a negative example to the Baues question, for three reasons: firstly, a triangulation with no flips at all would be an isolated element in the refinement poset, thus providing such an example; secondly, triangulations with very few flips are nodes of very low order in the graph of triangulations of a point configuration, which increase the chances of the graph being disconnected; thirdly, the cases mentioned 
above in which the graph of triangulations is known to be connected are more or less the same ones for which triangulations are known not to have flip-deficiency. Exceptions to this rule are the case of cyclic polytopes already mentioned and that de Loera et al. [8] have proved that triangulations in dimension 3 with all the vertices in convex position have no flip-deficiency, while the connectivity question is not settled in this case.

We mention also that another paper by the author [20] shows that when the notions of triangulation and bistellar flip are generalized in the natural way to triangulations of oriented matroids, there is a triangulation of a nonrealizable acyclic polytopal rank 34 oriented matroid on 38 elements with no flips. This can be considered a combinatorial analogue of a triangulation with no flips of a 33-dimensional polytope with 38 vertices. This uses a construction of Richter-Gebert [19].

\section{Flips and Vector Configurations}

Throughout the paper we work in the framework of simplicial fans of vector configurations which is more general than that of triangulations of point configurations. This is essentially the same approach as in [3].

Let $\mathcal{A}$ be a finite subset of the real vector space $\mathbb{R}^{d+1}$ (this is what we call a vector configuration of dimension $d$ ). A cone of $\mathcal{A}$ is the positive span $\operatorname{pos}(\sigma)$ of a subset $\sigma$ of $\mathcal{A}$. If $\sigma$ is linearly independent we say that the cone is simplicial. A face of a simplicial cone $\operatorname{pos}(\sigma)$ is the simplicial cone $\operatorname{pos}(\tau)$ generated by any subset $\tau$ of $\sigma$. A facet of $\operatorname{pos}(\sigma)$ is a maximal proper face of it. A simplicial fan of $\mathcal{A}$ is a collection $T$ of full-rank simplicial cones of $\mathcal{A}$ such that the intersection $\operatorname{pos}\left(\sigma_{1}\right) \cap \operatorname{pos}\left(\sigma_{2}\right)$ of any two simplicial cones of $T$ is a face of both (possibly the face $\{0\}=\operatorname{pos}(\emptyset)$ ) and that $\bigcup_{\operatorname{pos}(\sigma) \in T} \operatorname{pos}(\sigma)=\operatorname{pos}(\mathcal{A})$.

Scaling the vectors of a configuration by positive scalars does not affect what simplicial fans can be obtained. Hence we can consider all the vectors to lie in the unit sphere $S^{d} \subset \mathbb{R}^{d+1}$. In this setting, simplicial fans become simplicial complexes geometrically realized in the sphere by geodesic simplices which use the points of $\mathcal{A}$ as vertices and cover the convex hull of $\mathcal{A}$ (where the convex hull of $\mathcal{A}$ in the sphere is taken in an obvious natural way: it is the intersection of the sphere with the positive span of $\mathcal{A}$ ). For this reason, and in order to unify the nomenclature, throughout this paper we use the term triangulation of a vector configuration meaning simplicial fan and convex hull meaning positive span. We also apply the terms link, star, or join in a triangulation of a vector configuration with the meaning they would have in the associated simplicial complex (see [10]).

We say that $\mathcal{A}$ is totally cyclic (or complete) if it positively spans $\mathbb{R}^{d+1}$. We say that $\mathcal{A}$ is acyclic (or pointed) if there is a linear functional $h$ which is positive on every point of $\mathcal{A}$. In this case the vector configuration $\mathcal{A}$ is equivalent for the purpose of triangulations to the configuration $\{a / h(a) \mid a \in \mathcal{A}\}$, which is a point configuration of dimension $d$ in the affine plane $h^{-1}(1)$. Reciprocally, a point configuration $\mathcal{A} \subset \mathbb{R}^{d}$ can be regarded as a vector configuration $\mathcal{A}^{\prime} \in \mathbb{R}^{d+1}$, identifying the affine space $\mathbb{R}^{d}$ with an affine nonlinear hyperplane in $\mathbb{R}^{d+1}$. In this sense the concept of triangulation (simplicial fan) of a vector configuration is a generalization of the concept of triangulation of a point configuration.

Following the terminology of matroid theory, we call a minimal linearly dependent subset of $\mathcal{A}$ a circuit (see [5] or [21] for details). The unique (up to a scalar factor) depen- 
dence equation in a circuit divides its elements into two parts $Z=Z_{+} \cup Z_{-}$containing respectively the elements with positive and negative coefficient in the equation. The pair $\left(Z_{+}, Z_{-}\right)$is called an oriented circuit; of course, if $\left(Z_{+}, Z_{-}\right)$is an oriented circuit, then so is $\left(Z_{-}, Z_{+}\right)$, and the two of them are the only orientations of $Z=Z_{+} \cup Z_{-}$. Since our interest will always be in oriented circuits we use the word circuit assuming they have an orientation and call the underlying unoriented circuit $Z$ the support of $\left(Z_{+}, Z_{-}\right)$.

We say that a circuit is acyclic if both $Z_{+}$and $Z_{-}$are nonempty (equivalently if its support $Z$ is an acyclic vector configuration). In a point configuration, all the circuits are acyclic and they coincide with the so-called minimal Radon partitions; that is to say, minimal pairs $\left(Z_{+}, Z_{-}\right)$such that the relative interiors of $\operatorname{conv}\left(Z_{+}\right)$and $\operatorname{conv}\left(Z_{-}\right)$ intersect in a point. The support $Z$ of an acyclic circuit $\left(Z_{+}, Z_{-}\right)$can be triangulated in exactly two ways:

$$
T_{+}(Z):=\left\{\operatorname{conv}(Z-\{p\}) \mid p \in Z_{+}\right\} \quad T_{-}(Z)=\left\{\operatorname{conv}(Z-\{p\}) \mid p \in Z_{-}\right\} .
$$

Definition 1. Let $T$ be a triangulation of $\mathcal{A}$ and let $\left(Z_{+}, Z_{-}\right) \subset \mathcal{A}$ be a circuit of $\mathcal{A}$. Suppose that the following conditions are satisfied:

(i) The triangulation $T_{+}(Z)$ is a subcomplex of $T$.

(ii) All the maximum-rank simplices of $T_{+}(Z)$ have the same link $L$ in $T$. In particular, $T_{+}(Z) * L$ is a subcomplex of $T$. Here and in what follows we denote by $A * B$ the join of two simplicial complexes $A$ and $B[10]$.

In these conditions we can obtain a new triangulation $T^{\prime}$ of $\mathcal{A}$ by replacing the subcomplex $T_{+}(Z) * L$ of $T$ with the complex $T_{-}(Z) * L$. This operation of changing the triangulation is called a geometric bistellar flip (or a flip, for short) supported on the circuit $\left(Z_{+}, Z_{-}\right)$. We say that $T$ and $T^{\prime}$ are geometric bistellar neighbors. We call the flip of type $(k, l)$ if $Z_{+}$and $Z_{-}$have $k$ and $l$ elements, respectively.

Observe that our definition of flip supported on a circuit explicitly assumes that the circuit is oriented so that the star of the negative part of the circuit is "flipped out" and the positive part is "flipped in." This convention (which is not present in other definitions, see page 231 of [9]) is important in our exposition.

When counting the number of flips of a triangulation in Sections 3 and 4 the properties that we state as lemmas below will be helpful. Let $\operatorname{conv}\left(\sigma_{1}\right)$ and $\operatorname{conv}\left(\sigma_{2}\right)$ be two maximal simplices of a triangulation $T$ which share a facet $\operatorname{conv}(\tau)$. Let $a_{1}$ and $a_{2}$ be the two vertices joined to $\tau$; that is to say, let $\sigma_{1}=\tau \cup\left\{a_{1}\right\}$ and $\sigma_{2}=\tau \cup\left\{a_{2}\right\}$. Then $\rho=\tau \cup\left\{a_{1}, a_{2}\right\}$ is a spanning set of $\mathcal{A}$ with $d+1$ elements, and there is a unique circuit $\left(Z_{+}, Z_{-}\right)$with support contained in $\rho$, oriented so that $a_{1}, a_{2} \in Z_{+}$. We say that the facet $\tau$ is flippable if there is a flip supported on that circuit. If we assume that all the vectors/points of $\mathcal{A}$ are used as vertices of $T$, then no flips of type $(1, k)$ are possible and every flip of $T$ has one or more flippable facets. Reciprocally, a flippable facet can only correspond to one flip. That is to say:

Lemma 2. In a triangulation which uses all the elements as vertices, an exhaustive search of flippable facets gives all the possible flips. 
Given a vector configuration $\mathcal{A} \in \mathbb{R}^{d+1}$ and a nonzero vector $a \in \mathcal{A}$, we call the contraction of $\mathcal{A}$ at $a_{1}$ and denote $\mathcal{A} / a_{1}$ as the vector configuration $\left\{v-\left(\left(a_{1} \cdot v\right) /\left(a_{1}\right.\right.\right.$. $\left.\left.\left.a_{1}\right)\right) a_{1} \mid v \in \mathcal{A} \backslash\left\{a_{1}\right\}\right\} . \mathcal{A} / a_{1}$ can be considered a vector configuration in one dimension less since all its vectors are orthogonal to $a_{1}$. For a point configuration $\mathcal{A} \in \mathbb{R}^{d}$, the contraction at a point $a_{1} \in \mathcal{A}$ is the vector configuration $\mathcal{A} / a_{1}=\left\{b-a_{1} \mid b \in\right.$ $\left.\mathcal{A} \backslash\left\{a_{1}\right\}\right\} \subset \mathbb{R}^{d}$.

Lemma 3. Let $T$ be a triangulation of a point or vector configuration $\mathcal{A}$. Let $a_{1}$ be a vertex of one of the simplices of $T$ which are removed by a flip on a certain circuit $\left(Z_{+}, Z_{-}\right)$. Then:

(i) $\left(Z_{+} \backslash\left\{a_{1}\right\}, Z_{-} \backslash\left\{a_{1}\right\}\right)$ is a circuit of $\mathcal{A} / a_{1}$.

(ii) link $\left(a_{1}\right)$ is a triangulation of the vector configuration $\mathcal{A} / a_{1}$.

(iii) link $\left(a_{1}\right)$ has a flip supported on the circuit $\left(Z_{+} \backslash\left\{a_{1}\right\}, Z_{-} \backslash\left\{a_{1}\right\}\right)$.

Proof. Easy. Left to the reader.

\section{A Construction in Dimension 3}

Let $n$ be an even positive integer. Throughout this section we call $\mathcal{A}_{n}$ the following point configuration with $n^{2}+2 n+2$ points in $\mathbb{R}^{3}$ :

- The $n^{2}+2$ points $(x, y, 0) \in \mathbb{R}^{3}$ in the plane $z=0$ whose coordinates $x$ and $y$ are integer and satisfy

$$
\begin{gathered}
|x| \leq 2 y \leq 2 n+1-|x|, \\
2 y \neq|x|+1, \quad 2 y \neq 2 n-|x| .
\end{gathered}
$$

We call these points the planar part of $\mathcal{A}_{n}$. Figure 1 shows a triangulation of the planar part of $\mathcal{A}_{n}$ (for $n=12$ ) which will be used later.

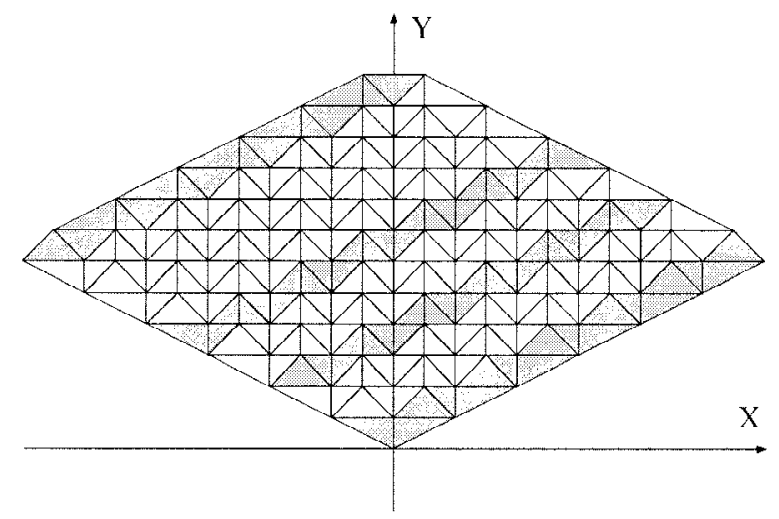

Fig. 1. A triangulation of the planar part of $\mathcal{A}_{12}$. 
- The $n$ points $P_{i}:=(0, i, 1)$, for $i=1, \ldots, n$, "above" the planar part.

- The $n$ points $Q_{i}:=(0, i,-1)$, for $i=1, \ldots, n$, "below" the planar part.

The triangulation in Fig. 1 is obtained by considering all the possible triangles in the configuration of the form $[(x, y, 0),(x+1, y+1,0),(x, y+1,0)]$ and $[(x, y, 0),(x+$ $1, y+1,0),(x+1, y, 0)]$ for even $x$, all the triangles of the form $[(x, y, 0),(x+$ $1, y, 0),(x, y+1,0)]$ and $[(x+1, y+1,0),(x, y+1,0),(x+1, y, 0)]$ for odd $x$, and then completing with the introduction of the segments $[(0,0,0),(0,1,0)],[(n-$ $2, n / 2,0),(n-1, n / 2+1,0)]$ and $[(-n+2, n / 2,0),(-n+1, n / 2+1,0)]$ and the segments $[(x-1, y, 0),(x+1, y, 0)]$ for every "missing" integer point $(x, y, 0)$ with $2 y=|x|+1$ or $2 y=2 n-|x|$. Our goal is to construct a triangulation of $\mathcal{A}_{n}$ which extends this one and show that it has $O(n)$ flips.

We proceed as follows: for each $i=1, \ldots, n$, we join $P_{i}$ to all the triangles in the planar part which have their vertices in the diagonal strip $x+2 i-2 \leq 2 y \leq x+2 i+1$. The four shaded regions in Fig. 1 show the triangles to be joined to $P_{1}, P_{4}, P_{7}$, and $P_{12}$, respectively. Then we insert tetrahedra of the form $\left[P_{i}, P_{i+1}, P, Q\right]$, where $[P, Q]$ are certain edges of the triangulation of the planar part, in order to "fill in the gaps." This can be done in a unique way to provide a triangulation of the upper half $\mathcal{A}_{n} \backslash\left\{Q_{1}, \ldots, Q_{n}\right\}$ of $\mathcal{A}_{n}$.

Finally, we triangulate the lower half by applying the symmetry transformation $[x \rightarrow$ $-x ; z \rightarrow-z]$. In other words, we consider diagonal strips in the planar part in the other possible direction and join them to the points $Q_{1}, \ldots, Q_{n}$. Let $T_{n}$ be the triangulation of $\mathcal{A}_{n}$ obtained in this way.

In order to clarify the construction Fig. 2 shows what we get as the link of each $P_{i}$, $i=1, \ldots, n$ (for $n=6$ ). Upward arrows in the link of $P_{i}$ represent edges going to
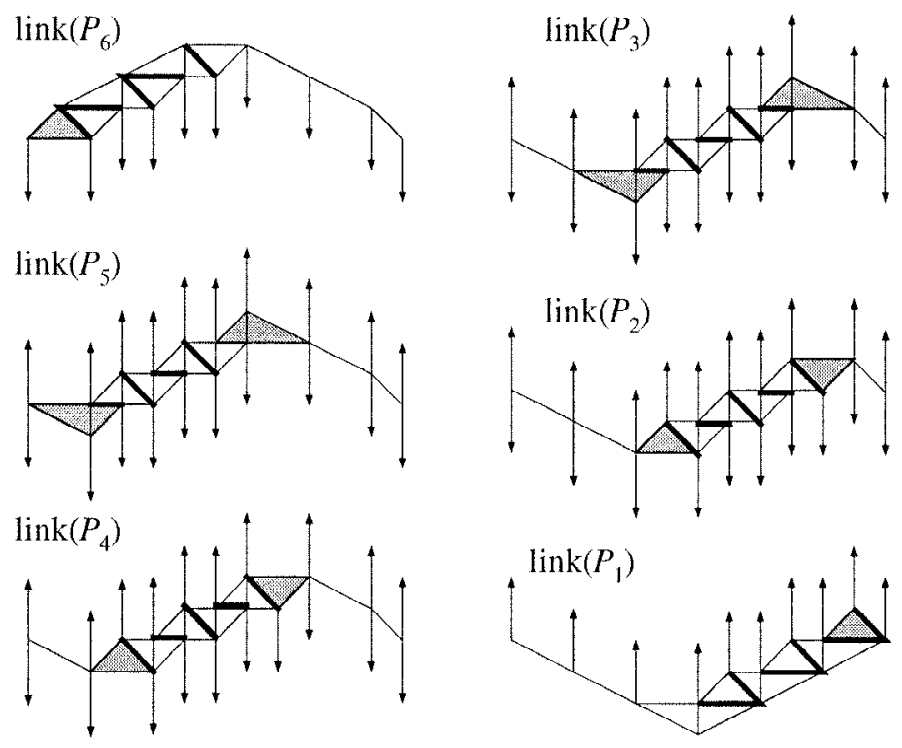

Fig. 2. Links of the points $P_{i}$ in the triangulation of $\mathcal{A}_{6}$. 
$P_{i+1}$ and downward arrows represent edges going to $P_{i-1}$. In particular, there will be an edge $\left[P_{i}, P_{i+1}\right]$ for $i=1, \ldots, n-1$. The link of $Q_{i}$ is obtained from that of $P_{i}$ by the symmetry $[x \rightarrow-x ; z \rightarrow-z]$. The links of $P_{i}$ and $Q_{i}, i=1, \ldots, n$, completely characterize the triangulation, since every tetrahedron contains one of those points as a vertex. Thick edges in the figure separate parts of the link of a point $P_{i}$ which are joined to different points among the $Q_{j}$ 's. The shaded parts indicate some of the flips of the triangulation, to be discussed now:

(i) Suppose that a flip is supported in a circuit fully contained in the planar part. This has to correspond to a flip in Fig. 1 but, moreover, all the triangles of the planar part which disappear by the flip have to be joined to the same points $P_{i}$ and $Q_{j}$. In other words, the planar flip has to be contained in one of the links of Fig. 2 and the triangles involved not be separated by a thick edge. A quick look at Fig. 2 shows that there are no flips of this type.

(ii) Suppose that a flip is supported in a circuit $C$ that contains a point $P_{i}$ and no $Q_{j}$. Since the circuits of type $\left(\left\{P_{i-1}, P_{i+1}\right\},\left\{P_{i}\right\}\right)$ are clearly nonflippable, $C$ must contain exactly two consecutive points $P_{i}$ and $P_{i+1}$ and either two or three points in the planar part.

If $C$ contains two points in the planar part, they are of the form $(x, y, 0)$ and $(x, y+1,0)$. In this case the flip corresponds to moving the two triangles incident to the edge $[(x, y, 0),(x, y+1,0)]$ up (from $\operatorname{link}\left(P_{i}\right)$ to $\left.\operatorname{link}\left(P_{i+1}\right)\right)$ or down (from $\operatorname{link}\left(P_{i+1}\right)$ to $\left.\operatorname{link}\left(P_{i}\right)\right)$.

For example, the two shaded triangles in the right-top corner of $\operatorname{link}\left(P_{3}\right)$ in Fig. 2 represent a flip in the circuit $\left(\left\{P_{3},(3,5,0)\right\},\left\{P_{4},(3,4,0)\right\}\right)$. The triangles $\left[P_{3}, P_{4},(3,4,0)\right]$ and $\left[(3,5,0), P_{4},(3,4,0)\right]$ are both present in the triangulation and have the same link, consisting of the points $(2,4,0)$ and $(5,4,0)$. After performing the flip in this circuit, the links drawn in Fig. 2 would change in that the two shaded triangles $[(3,5,0),(3,4,0),(2,4,0)]$ and $[(3,5,0),(3,4,0),(5,4,0)]$ will now appear in $\operatorname{link}\left(P_{4}\right)$ instead of $\operatorname{link}\left(P_{3}\right)$ (with the corresponding changes in the upward and downward arrows). This is what we call "moving up" two triangles and a similar "moving down" will be possible with the two shaded triangles in the bottom-left corner of $\operatorname{link}\left(P_{3}\right)$.

Looking at Fig. 2 one checks that there are exactly $n-2$ flips of this type, two in $\operatorname{link}\left(P_{i}\right)$ for each odd $i=3,5, \ldots, n-1$.

If $C$ contains three points in the planar part, then these three points are the vertices of a triangle and the flip corresponds to moving this triangle up or down in a similar fashion. There are $n$ flips of this type: two in $\operatorname{link}\left(P_{i}\right)$ for each even $i=2,4, \ldots, n-2$ plus one in $\operatorname{link}\left(P_{1}\right)$ and one in $\operatorname{link}\left(P_{n}\right)$.

(iii) By symmetry, we conclude that there are also $n-2+n=2 n-2$ flips in circuits containing one of the points $Q_{j}$ and none of the $P_{i}$.

(iv) Let $\left(Z_{+}, Z_{-}\right)$be a circuit which supports a flip and which contains at least one $P_{i}$ and one $Q_{j}$. Since $Z_{+} \cup Z_{-} \backslash\{a\}$ is a simplex in the triangulation for every $a \in Z_{+}$and since $P_{i}$ and $Q_{j}$ are not joined by an edge, $Z_{+} \subset\left\{P_{i}, Q_{j}\right\}$. In fact, $Z_{+}=\left\{P_{i}, Q_{j}\right\}$ because otherwise the unique point in $Z_{+}$should lie in the relative interior of the convex hull of the simplex $Z_{-} \in T$, and thus the point in $Z_{+}$would not be used in the triangulation, which is not the case. Since $Z_{-} \cup\left\{P_{i}\right\}$ 
and $Z_{-} \cup\left\{Q_{j}\right\}$ are simplices in the triangulation, $Z_{-}$has to be the set of vertices of a simplex in the planar part of $T$, and the relative interior of this simplex intersects the segment $\left[P_{i}, Q_{j}\right]$. In particular, the point $(0,(i+j) / 2,0)$ must lie in the relative interior of $\operatorname{conv}\left(Z_{-}\right)$. Then:

- If $i+j$ is odd, we call $k=(i+j+1) / 2$. We must have $Z_{-}=\{(0, k-$ $1,0),(0, k, 0)\}$. Since the segment $[(0, k-1,0),(0, k, 0)]$ is joined in $T_{n}$ to the points $P_{k}$ and $Q_{k}$ and to no other of the $P_{l}$ 's or $Q_{l}$ 's, we conclude that $i=j=k$, which contradicts the fact that $i+j$ is odd.

- If $i+j$ is even, we call $k=(i+j) / 2$. Then $Z_{-}=\{(0, k, 0)\}$. This point is joined to $P_{k}, P_{k+1}, Q_{k}$, and $Q_{k+1}$, and to no other $P_{l}$ or $Q_{l}$. Thus, in order to have $i+j=2 k$ we must have $i=j=k$ and the circuit must be $\left(\left\{P_{k}, Q_{k}\right\},\{(0, k, 0)\}\right)$. For this circuit to be flippable, the segments $\left[P_{k},(0, k, 0)\right]$ and $\left[Q_{k},(0, k, 0)\right]$ need to have the same link, and this only happens for $k=n$. Thus, there is only one flip of this type.

Summarizing, we conclude that:

Theorem 4. The point configuration $\mathcal{A}_{n} \subset \mathbb{R}^{3}$ has $n^{2}+2 n+2$ points. The triangulation $T_{n}$ of $\mathcal{A}_{n}$ has $4 n-3$ flips.

\section{Stacking Layers of Prisms}

\section{Prisms}

Our construction in dimension 4 will be based on the strategy of stacking several layers of triangulated prisms in a concentric way without increasing the number of flips. A similar technique has implicitly been used in the last construction in [8].

The standard $d$-dimensional prism is the point configuration $\left\{e_{1}, \ldots e_{d}, e_{1}+e_{0}, \ldots\right.$, $\left.e_{d}+e_{0}\right\} \subset \mathbb{R}^{d+1}$, where $e_{0}, e_{1}, \ldots, e_{d}$ is an affine basis in $\mathbb{R}^{d}$. We say that a (vector or point) configuration $\mathcal{A}$ is a $d$-prism if it consists of $2 d+2$ points and there is a bijective correspondence between them and the points in the standard prism which preserves circuits (in other words, if $\mathcal{A}$ has the same oriented matroid as the standard prism). That is to say, the configuration $\mathcal{A}=\{0, \ldots, d, \overline{0}, \ldots, \bar{d}\}$ is a prism if its circuits are the pairs of the form $(\{i, \bar{j}\},\{j, \bar{i}\})$, for $i \neq j$. Any prism is projectively equivalent to the standard one.

The subsets $\{0, \ldots, d\}$ and $\{\overline{0}, \ldots, \bar{d}\}$ of a prism are the vertices of two simplicial facets of the prism. We call these facets the floor and the ceiling of the prism respectively, although which of them is the floor and which the ceiling is an arbitrary choice. Triangulations of a $d$-dimensional prism are in bijective correspondence with permutations of the symbols $[0, \ldots, d]$ via the following rule: to the permutation $\left[i_{0}, \ldots, i_{d}\right]$ we associate the triangulation

$$
\left\{\operatorname{conv}\left(\left\{i_{0}, \ldots, i_{l}, \bar{i}_{l}, \ldots, \overline{i_{d}}\right\}\right) \mid l=0, \ldots, d\right\} .
$$

Moreover, two such triangulations differ by a bistellar flip if and only if the corresponding permutations differ by a transposition. This correspondence is well known (see Section 7.3 of [9]) and makes the secondary polytope of the prism a permutahedron. 
On the other hand, permutations of $[0, \ldots, d]$ correspond in an obvious bijective way with acyclic orientations of the complete graph $K_{d+1}$ on $d+1$ vertices: we identify the nodes of $K_{d+1}$ with the $d+1$ symbols $0, \ldots, d$ and orient the edges in $K_{d+1}$ according to the total order in the vertices given by the permutation. Transpositions in the permutation correspond to reversing a single edge in the graph. Summing up:

Lemma 5. Let $\tau \cup \bar{\tau}$ be a prism with floor $\tau$ and ceiling $\bar{\tau}$. Then the triangulations of the prism are in bijective correspondence with acyclic orientations of the 1-skeleton of $\operatorname{conv}(\tau)$ and two triangulations differ by a bistellar flip if and only if the corresponding orientations of the 1-skeleton differ by reorientation of a single edge.

A further observation will be used later. In the conditions of the statement, let $i_{0}, i_{d} \in \tau$ respectively be the unique source and sink in a given acyclic orientation of the 1-skeleton of $\operatorname{conv}(\tau)$. In the triangulation of the prism corresponding to that orientation, the floor $\operatorname{conv}(\tau)$ of the prism is joined to the vertex $\bar{i}_{0}$ which is above the source $i_{0}$ and the ceiling $\bar{\tau}$ is joined to the sink $i_{d}$. Also, all the facets of the prism other than the ceiling and the floor are prisms over facets of $\operatorname{conv}(\tau)$ and the restriction of $T$ to them is obtained by restricting the orientation chosen in the 1 -skeleton of $\operatorname{conv}(\tau)$ to that facet.

Triangulating Layers of Prisms. Let $T$ be a triangulation of a point or vector configuration $\mathcal{A}$ and let $O \in \mathcal{A}$ be a distinguished vertex. Let $\mathcal{B}$ be the subconfiguration of $\mathcal{A}$ consisting of the vertices in the link of $O$ and let $\mathcal{C}$ be the subconfiguration of vertices not in the star of $O$ (so that $\mathcal{A}$ is the disjoint union of $\{O\}, \mathcal{B}$ and $\mathcal{C}$ ). In the case of a vector configuration, we assume further that $\{O\} \cup \mathcal{B}$ is acyclic and without loss of generality we consider $\{O\} \cup \mathcal{B}$ to lie in an affine hyperplane.

Let $c$ be a positive real number smaller than 1 (we typically take $c$ to be very close to 1). For each integer $n$, consider the following (point or vector) configurations:

$$
\mathcal{B}_{n}:=\left\{c^{n} P+\left(1-c^{n}\right) O ; P \in \mathcal{B}\right\}, \quad \mathcal{A}_{n}:=\{O\} \cup \mathcal{B}_{0} \cup \cdots \cup \mathcal{B}_{n} \cup \mathcal{C} .
$$

That is, $\mathcal{B}_{0}=\mathcal{B}$ and $\mathcal{B}_{n}$ is a copy of $\mathcal{B}_{n-1}$ contracted toward $O ; \mathcal{A}_{n}$ is obtained adding the $n$ contracted copies of $\mathcal{B}$ to $\mathcal{A}$. We denote by $c^{n} \tau$ and $c^{n} a$ the images in $\mathcal{B}_{n}$ of any subset $\tau$ or element $a$ of $\mathcal{B}$. This is a slight abuse of notation unless $O$ is taken as the origin of coordinates (which can be done for a point configuration but not for a vector configuration).

In these conditions, the following is a polytopal subdivision (a fan in the standard terminology for vector configurations) of $\mathcal{A}_{n}$ : the antistar of $O$ in $T$ together with, for each simplex $\operatorname{conv}(\tau)$ of $T$ in the link of $O$, the simplex $\operatorname{conv}\left(\{O\} \cup c^{n} \tau\right)$ and the cells $\operatorname{conv}\left(c^{k} \tau \cup c^{k-1} \tau\right)$ for each $k=1, \ldots, n$. Observe that what we do is contracting the star of $O$ in $T$ and inserting the cells $\operatorname{conv}\left(c^{k} \tau \cup c^{k-1} \tau\right)$ in between the star and the antistar. Moreover, the cells $\operatorname{conv}\left(c^{k} \tau \cup c^{k-1} \tau\right)$ that we have introduced are (convex hulls of) prisms, whose floor and ceiling are contracted copies of the simplices in the link of $O$ in $T$. Figure 3 shows an example of the construction.

The subdivision $S_{n}$ described above is called the subdivision obtained by inserting $n$ layers of prisms around $O$ in $T$ with parameter $c$. We call the $k$ th layer of $S_{n}$, for $k=1, \ldots, n$, the union of the prisms $\operatorname{conv}\left(c^{k} \tau \cup c^{k-1} \tau\right)$. 

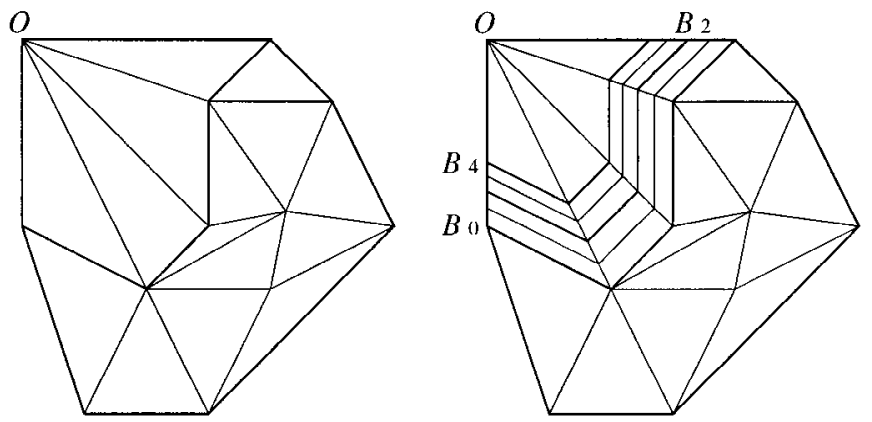

Fig. 3. Insertion of four layers of prisms in a planar triangulation.

If we want to refine $S_{n}$ into a triangulation, we just need to triangulate each of the prisms in the layers. We concentrate on a layer with index $k$. The prisms in the layer have a simplicial facet in $\mathcal{B}_{k-1}$ and another one in $\mathcal{B}_{k}$. We consider the first one to be the floor of the prism. In order to specify a triangulation of the layer we just need to give an orientation to all the edges of $S_{n}$ which have their vertices in $\mathcal{B}_{k-1}$ in such a way that each simplex is oriented acyclically. Figure 4 shows how to triangulate the third layer in the example of Fig. 3.

Since the floor of each layer is a contracted version of the link of $O$ in the original triangulation $T$, we conclude that:

Lemma 6. Let $S_{n}$ be the subdivision obtained by inserting $n$-layers of prisms around the vertex $O$ in a certain triangulation $T$. Let $G$ be the 1-skeleton of the link of $O$ in $T$. Then in order to refine $S_{n}$ into a triangulation it is sufficient to choose $n$ oriented copies $G_{1}, \ldots, G_{n}$ of the graph $G$, all of them acyclic on every simplex of the link, and use $G_{i}$ to triangulate the $i$ th layer, for $i=1, \ldots, n$.

\section{Inserting Layers with No Addition of Flips}

Remember that flips in triangulations of a prism correspond to edges which can be reversed in the orientation of the floor; then it will be interesting to us that the orientations
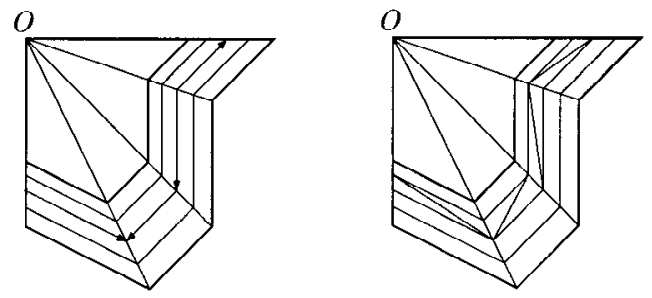

Fig. 4. Orienting the edges in $\mathcal{B}_{2}$ we triangulate a layer of Fig. 3. 
$G_{1}, \ldots, G_{n}$ which appear in Lemma 6 be "rigid" in the following sense:

Definition 7. Let $K$ be a simplicial complex and let $G$ be its 1 -skeleton. We say that an orientation of $G$ is rigid (with respect to $K$ ) if it is acyclic on every simplex of $K$ but reversing the orientation of any single edge makes it cyclic in some simplex. Following standard graph theory terminology we say that the orientation has no sources or sinks if no vertex of $G$ has all the orientations of its incident edges in-going or out-going.

For a directed graph $G,-G$ denotes the opposite orientation of $G$.

Theorem 8. Let $T$ be a triangulation of a configuration $\mathcal{A}$ which uses all the elements. Let $O \in \mathcal{A}$ be one of the vertices of the triangulation and let $L$ be the link of $O$ in $T$. Suppose that no two adjacent maximal simplices in L lie in the same hyperplane (we say that the link of $O$ is generic when it satisfies this condition). Let $S_{n}$ be the subdivision obtained by inserting $n$ layers around $O$, with the parameter $c$ which appears in the construction sufficiently close to 1.

Let $G$ be a rigid orientation of the 1-skeleton of $L$ with no sources or sinks. Consider the refinement $T_{n}$ of $S_{n}$ obtained as in Lemma 6 using $G_{k}=G$ for even $k$ and $G_{k}=-G$ for odd $k$. Then:

(i) $T_{n}$ has exactly the same number of flips as $T_{1}$, for every $n$.

(ii) For every flip of $T_{n}$, either all the flippable facets contain $O$ or some flippable facet has its vertices contained in $\mathcal{B}_{0} \cup \mathcal{C}$.

Proof. Recall that we can count flips of $T_{n}$ by saying which facets of $T_{n}$ are flippable, as in Lemma 2 (we call facets of $T_{n}$ the facets of the maximal cells of $T_{n}$ ).

Let $\tau$ be a facet in $T_{n}$. By construction, either $\tau$ contains $O$, or is contained in $\mathcal{B}_{0} \cup \mathcal{C}$, or is contained in $\mathcal{B}_{k} \cup \mathcal{B}_{k-1}$, for some $k \in\{1, \ldots, n\}$. The following cases (a)-(d) cover all possibilities except for the case $\tau \subset \mathcal{B}_{0} \cup \mathcal{C}$ which we do not need to care about. The four cases are illustrated in parts (a)-(d) of Fig. 5, where the facets under study are drawn thicker:

(a) Let $\operatorname{conv}\left(c^{n} \tau \cup\{O\}\right)$ be a facet of $T_{n}$ which has $O$ as a vertex, and suppose that it is flippable. By construction of $T_{n}, \operatorname{conv}\left(c^{n} \tau \cup\{O\}\right)$ is the contracted version of a certain facet $\operatorname{conv}(\tau \cup\{O\})$ of $T$ with $\tau \subset \mathcal{B}_{0}=\mathcal{B}$, as is implicit in our notation. The facet $\operatorname{conv}(\tau)$ is joined in $T$ to at most two (perhaps one, but this case is treated in an analogous way) points $a$ and $b$ in $\mathcal{B}$, so that $\operatorname{conv}\left(c^{n} \tau \cup\{O\}\right)$ is joined to two points $c^{n} a$ and $c^{n} b$.

Since the link of $O$ in $T$ is generic, $O$ is in the support of the circuit contained in $\left\{c^{n} a, c^{n} b, O\right\} \cup c^{n} \tau$. Moreover, $\left\{c^{n} a, c^{n} b\right\} \cup c^{n} \tau$ is not a simplex in $T_{n}$ (since $\left\{c^{n} a\right\} \cup c^{n} \tau$ and $\left\{c^{n} b\right\} \cup c^{n} \tau$ are the ceilings of two different prisms in the $n$th layer). Then, for the circuit to be flippable (with our sign convention), it is necessary that $O$ be in the negative part of the circuit. This implies that all the maximal 


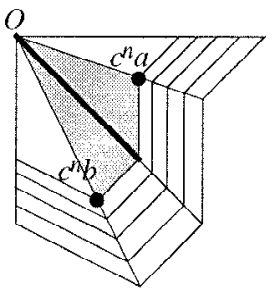

(a)

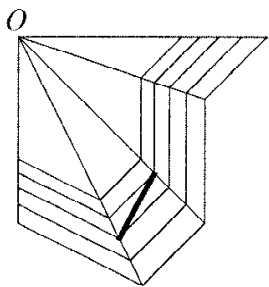

(c)

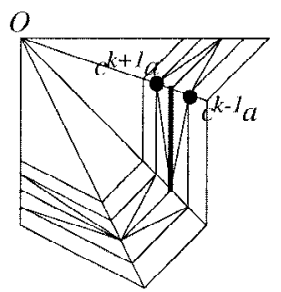

(b)

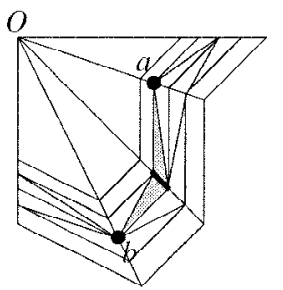

(d)

Fig. 5. Different types of facets in the proof of Theorem 8.

simplices to be removed by the flip (the shaded triangles in part (a) of Fig. 5) have $O$ as a vertex. In this case it is clear that the same flip can be performed in $T_{1}$ (actually, in $T$ as well), changing $c^{n} a, c^{n} b$, and $c^{n} \tau$ for $c^{1} a, c^{1} b$, and $c^{1} \tau$.

(b) We now see that no facet $\operatorname{conv}\left(c^{k} \tau\right)$ with $c^{k} \tau \subset \mathcal{B}_{k}(k \in\{1, \ldots, n\})$ is flippable. The facet $\operatorname{conv}\left(c^{k} \tau\right)$ is joined to a vertex $c^{k-1} a$ in $c^{k-1} \tau$ and either to $O$ (if $k=n$ ) or to an element $c^{k+1} b$ in $c^{n+1} \tau$, if $k<n$. More precisely, $a$ is the sink of $\tau$ in the orientation $G_{k}$ of the 1-skeleton of $L$ and, if $k<n, b$ is the source of $\tau$ in $G_{k+1}$. Since $G_{k}$ and $G_{k+1}$ are opposite to one another, $a=b$. If $\operatorname{conv}\left(c^{k} \tau\right)$ is flippable, the circuit in which the flip is supported is $\left(\left\{O, c^{n-1} a\right\},\left\{c^{n} a\right\}\right)$ if $k=n$ and $\left(\left\{c^{k-1} a, c^{k+1} a\right\},\left\{c^{k} a\right\}\right)$ if $k<n$. Thus, the link of $\operatorname{conv}\left(\left\{c^{k-1} a, c^{k} a\right\}\right)$ in $T_{n}$ has to coincide with that of $\operatorname{conv}\left(\left\{c^{k+1} a, c^{k} a\right\}\right)\left(\right.$ or $\operatorname{conv}\left(\left\{O, c^{n} a\right\}\right)$, if $\left.k=n\right)$ which implies that all the vertices in the link of $\operatorname{conv}\left(\left\{c^{k-1} a, c^{k} a\right\}\right)$ belong to $\mathcal{B}_{k}$. However, this would imply $a$ is a sink of the orientation $G_{k}$ of $L$. Since $G_{k}= \pm G$ and $G$ has no sources or sinks, $G_{k}$ has no sinks.

(c) Also, no facet interior to a prism $c^{k-1} \tau \cup c^{k} \tau$ can be flippable, because then the circuit involved must be one of the circuits of a prism. Performing the flip is equivalent to reversing the orientation of a certain edge in the graph $G_{k}$, which is impossible because $G_{k}$ is rigid.

(d) Finally, let $\operatorname{conv}(\tau)$ be a facet having vertices both in $\mathcal{B}_{k}$ and $\mathcal{B}_{k-1}$ for some $k \in\{1, \ldots, n\}$, but not interior to a prism. That is to say, the two maximal $\operatorname{simplices} \operatorname{conv}(\tau \cup a)$ and $\operatorname{conv}(\tau \cup b)$ sharing $\operatorname{conv}(\tau)$ belong to different prisms of the same layer. If the facet is flippable, the flip is supported in the unique circuit $Z=\left(Z_{+}, Z_{-}\right)$contained in $\tau \cup\{a, b\}$, oriented so that $\{a, b\} \subset Z_{+}$. The fact that no two facets in the link of $O$ in $T$ are coplanar implies that, if $c$ is sufficiently 
close to 1 , the segment $\operatorname{conv}(\{a, b\})$ is not contained in the $k$ th layer. We assume $c$ to be so close to 1 that this happens. Then:

(d.1) If $Z_{+}=\{a, b\}$, then this together with $Z_{-} \subset \tau$ implies that $\operatorname{conv}(\tau \cup\{a\})$ and $\operatorname{conv}(\tau \cup\{b\}$ ) (which are both contained in the $k$ th layer) cover $\operatorname{conv}\left(Z_{+} \cup Z_{-}\right)$. This contradicts the assumption that $\operatorname{conv}(\{a, b\})$ is not contained in the $k$ th layer.

(d.2) If there is an element $p \in Z_{+}$other than $a$ and $b$, then $\sigma=Z_{+} \cup Z_{-} \backslash\{p\}$ is a simplex of $T_{n}$ which contains $a$ and $b$. In particular, $a$ and $b$ are joined by an edge. The fact that $\operatorname{conv}(\{a, b\})$ is not contained in the $k$ th layer implies that this edge has not been introduced by the insertion of layers. Thus, the only possibility is that $k=1, a$ and $b$ are in $\mathcal{B}_{0}$, and $\sigma$ is a simplex outside the layers. In particular, $\sigma$ only contains vertices in $\mathcal{B}_{0} \cup \mathcal{C}$. The facets $\operatorname{conv}(\sigma \backslash\{a\})$ and $\operatorname{conv}(\sigma \backslash\{b\})$ of $\sigma$ are flippable by the flip, so that the flip is in the conditions of (ii).

The above case study implies that all the flips either are in the conditions of (a) or (d.2) or all their flippable facets lie in $\mathcal{B}_{0} \cup \mathcal{C}$. This proves part (ii) of the statement. The flips studied in (a) appear in $T_{1}$ as mentioned above. The flips mentioned in (d.2) and those with flippable facets in $\mathcal{B}_{0} \cup \mathcal{C}$ only remove simplices from the first layer or outside the layers. Thus, they are flips in $T_{1}$ as well. This proves (i).

Remark 9. Among the hypotheses in Theorem 8, the only one which is difficult to fulfill in a construction is a rigid orientation without sources or sinks of the 1-skeleton of $L$. If $\mathcal{A}$ is a configuration in dimension $d$, the link $L$ of a point is a triangulation of the $(d-1)$-sphere or the $(d-1)$-ball. It is clear that no orientation in a triangulation of the 1-sphere or 1-ball is rigid. For the 2-sphere or the 2-ball the same is not obvious, but still can be proved as follows.

Let $T$ be a triangulation of the 2-sphere or the 2-ball with $v$ vertices, $e$ edges, and $t$ triangles. Let an orientation be given to each edge in such a way that every triangle is acyclic. Then each triangle of $T$ prevents one of its edges being reversed, which implies that the number of edges whose orientations cannot be changed is at most $t$. Thus, in order for the orientation to be rigid we would need to have $t \geq e$. This is impossible since $t-e=\chi-v<0$ (where $\chi=1$ for the 2-ball and $\chi=2$ for the 2-sphere).

Still, a version of our construction can serve to insert layers of prisms in a threedimensional triangulation adding less flips per layer than vertices. The idea is to orient the 1-skeleton of a triangulated 2-sphere or 2-ball in a way acyclic on each triangle and with few reversible edges (edges whose reversal preserve acyclicness in triangles). The best possible ratio of reversible edges versus number of vertices is $3 / 5$, obtained by orienting the 1-skeleton of a triangular bipyramid (five vertices, six triangles) with only three reversible edges. Although it is impossible to do this without sinks or sources, this idea is used in [8] to obtain triangulations in dimension 3 with $5 n$ vertices and $3 n-2$ flips. 


\section{A Construction in Dimension Four}

\section{A Rigid Orientation of a Triangulation of the 3-Sphere}

We now show a rigid orientation with no sinks or sources of the 1-skeleton of a triangulation of the unit 3-sphere $S^{3} \subset \mathbb{R}^{4}$, in order to apply Theorem 8 to it.

Our triangulation will have 15 vertices that we denote $t_{i, j}, h_{i}$, and $v_{j}$ for $i, j=0,1,2$ (indices will be regarded modulo 3 in what follows), with coordinates

$$
\begin{gathered}
t_{i j}=\frac{1}{\sqrt{2}}\left(\cos \left(\frac{2 \pi i}{3}\right), \sin \left(\frac{2 \pi i}{3}\right), \cos \left(\frac{2 \pi j}{3}\right), \sin \left(\frac{2 \pi j}{3}\right)\right), \\
h_{i}=\left(\cos \left(\frac{2 \pi i}{3}\right), \sin \left(\frac{2 \pi i}{3}\right), 0,0\right), \quad v_{j}=\left(0,0, \cos \left(\frac{2 \pi j}{3}\right), \sin \left(\frac{2 \pi j}{3}\right)\right)
\end{gathered}
$$

(we use the letters $t, v$, and $h$ because if we think of $S^{3}$ in its stereographic projection $\Pi$ into $\mathbb{R}^{3}$ given by $\Pi(x, y, z, t)=(x /(t-1), y /(t-1), z /(t-1))$, then the points $h_{i}, t_{i, j}$, and $v_{j}$ lie, respectively, on a horizontal circle, a torus around it, and its vertical rotation axis).

The six points $h_{i}$ and $v_{j}$ are the vertices of the join $\Delta_{2} * \Delta_{2}$ of two triangles. The nine points $t_{i, j}$ are the vertices of the product $\Delta_{2} \times \Delta_{2}$ of two triangles. The common refinement of these two polytopes has 18 facets, all of which are triangular prisms: each of the six facets of $\Delta_{2} \times \Delta_{2}$ (which are 3-prisms themselves) is divided into three subprisms by its medial axis and each of the nine facets of $\Delta_{2} * \Delta_{2}$ (which are tetrahedra) is divided into two prisms by a quadrilateral with vertices in the midpoints of four edges.

Our triangulation $T$ of $S^{3}$ consists of the following 54 tetrahedra. For each $i=0,1,2$ and $j=0,1,2$ :

$$
\begin{array}{ccc}
{\left[h_{i}, t_{i, j}, t_{i, j+1}, t_{i+1, j+1}\right],} & {\left[h_{i}, t_{i, j}, t_{i+1, j}, t_{i+1, j+1}\right],} & {\left[h_{i}, h_{i+1}, t_{i+1, j}, t_{i+1, j+1}\right],} \\
{\left[v_{j}, t_{i, j}, t_{i+1, j}, t_{i+1, j+1}\right],} & {\left[v_{j}, t_{i, j}, t_{i, j+1}, t_{i+1, j+1}\right],} & {\left[v_{j}, v_{j+1}, t_{i, j+1}, t_{i+1, j+1}\right] .}
\end{array}
$$

Each of the two rows of tetrahedra above triangulates one of the 18 prisms mentioned, for each choice of indices $i$ and $j$. Since there are nine possible choices, this gives the total of 18 triangulated prisms.

The permutations $\left[t_{i, j} \rightarrow t_{i+1, j} ; h_{i} \rightarrow h_{i+1} ; v_{j} \rightarrow v_{j}\right]$ and $\left[t_{i, j} \rightarrow t_{j, i} ; h_{i} \rightarrow\right.$ $h_{i} ; v_{j} \rightarrow v_{j}$ ] produce symmetries of the triangulation $T$. They generate the symmetry group of the triangulation, which acts transitively and with trivial stabilizer over the 18 subprisms. Thus, the symmetry group has 18 elements. The first three tetrahedra in the list above are representatives for the three orbits of simplices.

There are two orbits of vertices in $T$, one containing all the $t_{i, j}$ and the other containing the $h_{i}$ and $v_{j}$. There are five orbits of edges. We show an orientation for a representative of each and let the others be oriented by the action of the symmetry group: we orient $\left[h_{i} \rightarrow h_{i+1}\right]$ (six edges in the orbit), $\left[t_{i, j} \rightarrow h_{i}\right]$ (18 edges), $\left[h_{i} \rightarrow t_{i+1, j}\right]$ (18 edges), $\left[t_{i, j} \rightarrow t_{i+1, j}\right]$ (18 edges), and $\left[t_{i, j} \rightarrow t_{i+1, j+1}\right]$ (nine edges). Then 
Theorem 10. The above is a rigid orientation of a triangulation of the 3-sphere with no sources or sinks. Thus, there are triangulations of point configurations in $\mathbb{R}^{4}$ and of vector configurations in $\mathbb{R}^{5}$ with an arbitrarily large number of vertices and a bounded number of flips.

Proof. The orientations shown for the edges produce the following acyclic orientations on the three representatives of simplices: $\left[t_{i, j} \rightarrow t_{i, j+1} \rightarrow h_{i} \rightarrow t_{i+1, j+1}\right],\left[t_{i, j} \rightarrow\right.$ $\left.h_{i} \rightarrow t_{i+1, j} \rightarrow t_{i+1, j+1}\right]$, and $\left[h_{i} \rightarrow t_{i+1, j} \rightarrow t_{i+1, j+1} \rightarrow h_{i+1}\right]$. Reorienting any of the five representatives of edges would produce a 3-cycle in a triangle of the triangulation; for example, the five triangles are: $\left[h_{i} \rightarrow t_{i+1, j} \rightarrow h_{i+1}\right],\left[t_{i, j} \rightarrow t_{i, j+1} \rightarrow h_{i}\right]$, $\left[h_{i} \rightarrow t_{i+1, j-1} \rightarrow t_{i+1, j}\right],\left[t_{i, j} \rightarrow h_{i} \rightarrow t_{i+1, j}\right]$, and $\left[t_{i, j} \rightarrow t_{i, j+1} \rightarrow t_{i+1, j+1}\right]$. Thus, the orientation is rigid. From the oriented cycles $\left[h_{0} \rightarrow h_{1} \rightarrow h_{2} \rightarrow h_{0}\right.$, $\left[v_{0} \rightarrow v_{1} \rightarrow v_{2} \rightarrow v_{0}\right]$, and $\left[t_{i, 0} \rightarrow t_{i, 1} \rightarrow t_{i, 2} \rightarrow t_{i, 0}\right], i=0,1,2$, it follows that no vertex is a source or a sink.

For the final statement, we consider the 15 vectors in $S^{3} \subset \mathbb{R}^{4}$ as points in $\mathbb{R}^{4}$ and consider the origin as an extra point. This gives a point configuration $\mathcal{A}$ with 16 points. For each tetrahedra in the sphere with vertices $\{a, b, c, d\}$ we consider the simplex with vertices $\{O, a, b, c, d\}$ in $\mathbb{R}^{4}$. This produces a collection of simplices of $\mathcal{A}$; we perturb a little the coordinates of every point so that the link of the origin $O$ is generic. This collection of simplices may not be a triangulation, if it does not cover the whole convex hull of $\mathcal{A}$. However, it can always be completed to a triangulation, perhaps with the addition of extra vertices. Thus, we can construct a triangulation of a point configuration $\mathcal{A}^{\prime}$ in $\mathbb{R}^{4}$ such that the link of one of the vertices is generic and admits a rigid orientation of edges without sinks or sources. Theorem 8 tells us how to construct triangulations with arbitrarily many vertices and a bounded number of flips from that.

\section{Counting Flips}

In what follows we look more closely at the above construction to compute an actual bound for the number of flips mentioned in the statement of Theorem 10 . Let $\mathcal{A}^{\prime} \subset \mathbb{R}^{5}$ be the following vector configuration: $\mathcal{A}^{\prime}:=\left\{O,-O, t_{i, j}, h_{i}, v_{j} \mid i, j=1,2,3\right\}$, where $O=(1,0,0,0,0),-O=(-1,0,0,0,0)$,

$$
\begin{aligned}
t_{i j} & =\frac{\alpha_{i, j}}{\sqrt{2}}\left(\frac{\sqrt{2}}{\alpha_{i, j}}, \cos \left(\frac{2 \pi i}{3}\right), \sin \left(\frac{2 \pi i}{3}\right), \cos \left(\frac{2 \pi j}{3}\right), \sin \left(\frac{2 \pi j}{3}\right)\right), \\
h_{i} & =\left(1, \alpha_{i} \cos \left(\frac{2 \pi i}{3}\right), \alpha_{i} \sin \left(\frac{2 \pi i}{3}\right), 0,0\right), \\
v_{j} & =\left(1,0,0, \alpha_{j} \cos \left(\frac{2 \pi j}{3}\right), \alpha_{j} \sin \left(\frac{2 \pi j}{3}\right)\right),
\end{aligned}
$$

for some positive coefficients $\alpha_{i, j}, \alpha_{i}$, and $\alpha_{j}(i, j=0,1,2)$.

The configuration $\mathcal{A}^{\prime}$ is obtained from $\mathcal{A}$ by scaling the 15 vectors of $\mathcal{A}$ with the coefficients $\alpha$, embedding $\mathbb{R}^{4}$ in an affine hyperplane of $\mathbb{R}^{5}$ with a central vector $O$, and including the opposite vector $-O$. Observe that $\mathcal{A}$ is (up to scaling) the contraction of $\mathcal{A}^{\prime}$ at $O$ (and at $-O$ as well). 
Let $T^{\prime}$ be the triangulation of $\mathcal{A}^{\prime}$ obtained by joining the triangulation $T$ of $\mathcal{A}$ to both $O$ and to $-O$, so that $\operatorname{link}(O)=\operatorname{link}(-O)=T$. The coefficients $\alpha_{i, j}, \alpha_{i}$, and $\alpha_{j}$ are chosen sufficiently generic for the link of $O$ in $T^{\prime}$ to satisfy the genericity condition required in the statement of Theorem 8 .

Proposition 11. The triangulation $T=\operatorname{link}(O)=\operatorname{link}(-O)$ has exactly nine flips, supported on the nine circuits of the form $\left(\left\{t_{i+1, j}, t_{i, j+1}\right\},\left\{t_{i, j}, t_{i+1, j+1}\right\}\right)$.

Proof. From the three representatives of simplices we conclude that there are six orbits of facets, with the following being representatives of them:

$$
\begin{array}{lr}
\operatorname{conv}\left(h_{i}, t_{i, j}, t_{i+1, j+1}\right), & \operatorname{conv}\left(h_{i}, t_{i, j}, t_{i+1, j}\right), \\
\operatorname{conv}\left(h_{i}, t_{i, j}, t_{i, j+1}\right), & \operatorname{conv}\left(h_{i}, t_{i+1, j}, t_{i+1, j+1}\right), \\
\operatorname{conv}\left(h_{i}, h_{i+1}, t_{i+1, j}\right), & \operatorname{conv}\left(t_{i, j}, t_{i, j+1}, t_{i+1, j+1}\right)
\end{array}
$$

We see that the last five facets are not flippable:

- the two vertices joined to $\operatorname{conv}\left(h_{i}, t_{i, j}, t_{i+1, j}\right)$ are $t_{i+1, j+1}$ and $t_{i, j-1}$. Since $2 t_{i, j}+$ $t_{i+1, j+1}+t_{i, j-1}=2 \sqrt{2} h_{i}+t_{i+1, j}$, the associated circuit is $\left(\left\{t_{i, j}, t_{i+1, j+1}, t_{i, j-1}\right\}\right.$, $\left.\left\{h_{i}, t_{i+1, j}\right\}\right)$. The circuit is not flippable, since $\operatorname{conv}\left(h_{i}, t_{i+1, j+1}, t_{i, j-1}, t_{i+1, j}\right)$ is not a simplex in the triangulation.

- the vertices joined to $\operatorname{conv}\left(h_{i}, t_{i, j}, t_{i, j+1}\right)$ are $h_{i-1}$ and $t_{i+1, j+1}$. Since $t_{i, j+1}=$ $t_{i+1, j+1}+(1 / \sqrt{2}) h_{i-1}+(2 / \sqrt{2}) h_{i}$, the associated circuit is $\left(\left\{t_{i, j+1}\right\},\left\{t_{i+1, j+1}\right.\right.$, $\left.\left.h_{i-1}, h_{i}\right\}\right)$. It is not flippable since $\operatorname{conv}\left(t_{i, j+1}, t_{i+1, j+1}, h_{i-1}\right)$ is not a simplex in the triangulation.

- the vertices joined to $\operatorname{conv}\left(h_{i}, t_{i+1, j}, t_{i+1, j+1}\right)$ are $h_{i+1}$ and $t_{i, j}$. Since $h_{i+1}+$ $\sqrt{2} t_{i, j}=h_{i}+\sqrt{2} t_{i+1, j}$ the associated circuit is $\left(\left\{t_{i, j}, h_{i+1}\right\},\left\{h_{i}, t_{i+1, j}\right\}\right)$, and it is not flippable since the links of $\operatorname{conv}\left(t_{i, j}, h_{i}, t_{i+1, j}\right)$ and $\operatorname{conv}\left(h_{i+1}, h_{i}, t_{i+1, j}\right)$ are not the same; the first one consists of the vertices $t_{i+1, j+1}$ and $t_{i, j-1}$ and the second one of $t_{i+1, j+1}$ and $t_{i+1, j-1}$.

- the two vertices joined to $\operatorname{conv}\left(h_{i}, h_{i+1}, t_{i+1, j}\right)$ are $t_{i+1, j+1}$ and $t_{i+1, j+2}$. The associated circuit is $\left(\left\{t_{i+1, j}, t_{i+1, j+1}, t_{i+1, j+2}\right\},\left\{h_{i+1}\right\}\right)$, since $t_{i+1, j}+t_{i+1, j+1}+$ $t_{i+1, j+2}=(3 / \sqrt{2}) h_{i+1}$. The link of $\operatorname{conv}\left(t_{i+1, j}, t_{i+1, j+1}, h_{i+1}\right)$ contains $t_{i+2, j+1}$ while the link of $\operatorname{conv}\left(t_{i+1, j}, t_{i+1, j+2}, h_{i+1}\right)$ contains $t_{i+2, j}$. Thus, the facet is not flippable.

- the vertices joined to $\operatorname{conv}\left(t_{i, j}, t_{i, j+1}, t_{i+1, j+1}\right)$ are $h_{i}$ and $v_{j}$. The associated circuit is $\left(\left\{t_{i, j}\right\},\left\{h_{i}, v_{j}\right\}\right)$ since $\sqrt{2} t_{i, j}=h_{i}+v_{j}$. It is not flippable since the links of $\operatorname{conv}\left(h_{i}, t_{i, j}\right)$ and $\operatorname{conv}\left(v_{j}, t_{i, j}\right)$ do not coincide; $h_{i-1}$ is a vertex in one but not in the other.

Thus, the only possible flippable facets are $\operatorname{conv}\left(h_{i}, t_{i, j}, t_{i+1, j+1}\right)$ and its images under the symmetry group. The vertices joined to this facet are $t_{i, j+1}$ and $t_{i+1, j}$ and the circuit is $\left(\left\{t_{i, j+1}, t_{i+1, j}\right\},\left\{t_{i, j}, t_{i+1, j+1}\right\}\right)$ since $t_{i, j+1}+t_{i+1, j}=t_{i, j}+t_{i+1, j+1}$. The circuit supports a flip since the facets $\operatorname{conv}\left(t_{i, j}, t_{i+1, j}, t_{i+1, j+1}\right)$ and $\operatorname{conv}\left(t_{i, j}, t_{i, j+1}, t_{i+1, j+1}\right)$ are joined to the same vertices, namely $h_{i}$ and $v_{j}$. 
Theorem 12. Inserting $n$ layers in the triangulation $T^{\prime}$ with a parameter $c$ sufficiently close to 1 gives a triangulation $T_{n}^{\prime}$ of a vector configuration in $\mathbb{R}^{5}$ with $15(n+1)+2$ vertices and nine flips.

Proof. By part (ii) of Theorem 8, all the flips of $T_{n}^{\prime}$ have a flippable facet containing $O$ or with its vertices in $\mathcal{B}_{0} \cup\{-O\}$.

Let $C$ be a circuit which produces a flip in which a flippable facet contains $O$. Genericity of the link of $O$ implies that $O$ is in the support of the circuit and that the rest of the elements in the circuit lie in the link of $O$, that is, they are in $\mathcal{B}_{n}$. In other words, $C$ is of the form $\left(c^{n} C_{+} \cup\{O\}, c^{n} C_{-}\right)$or $\left(c^{n} C_{+}, c^{n} C_{-} \cup\{O\}\right)$. The first case is impossible since then $\operatorname{conv}\left(c^{n} C_{+} \cup c^{n} C_{-}\right)$would have to be a simplex in our triangulation $T_{n}^{\prime}$, which is not the case. Thus, the flippable circuit is of the form $\left(c^{n} C_{+}, c^{n} C_{-} \cup\{O\}\right)$. Lemma 3 implies that $\left(c^{n} C_{+}, c^{n} C_{-}\right)$has to be a flippable circuit in $\operatorname{link}(O)$, considered as a triangulation of a vector configuration in $\mathbb{R}^{4}$. That is, $\left(c^{n} C_{+}, c^{n} C_{-}\right)$is the copy in $\mathcal{B}_{n}$ of one of the nine circuits of the configuration $\mathcal{A}$ which supports a flip of $T$. Thus, we conclude that the only circuits which can produce flips with flippable facets containing $O$ are those of the form $\left(c^{n} C_{+}, c^{n} C_{-} \cup\{O\}\right)$, where $\left(C_{+}, C_{-}\right)$is a flippable circuit of $T$. There are nine possibilities for $\left(C_{+}, C_{-}\right)$.

No flippable facet can have all its vertices in $\mathcal{B}_{0}$ : If $\operatorname{conv}\left(c^{0} \tau\right)$ is a flippable facet with its vertices in $\mathcal{B}_{0}$, then one of the vertices joined to $\operatorname{conv}\left(c^{0} \tau_{0}\right)$ is $-O$ and the other one is one of the vertices $c^{1} a$ of $c^{1} \tau$. Thus, the corresponding circuit is $\left(\left\{c^{1} a,-O\right\},\left\{c^{0} a\right\}\right)$. A flip supported on that circuit would imply that $a$ is a sink of the oriented graph used to construct the triangulation of the first layer, which is not the case.

Then part (ii) of Theorem 8 tells us that the remaining flips have a flippable facet containing $-O$. Genericity of the link of $O$ implies genericity of the link of $-O$. Thus, $-O$ is in the circuit which supports the flip and the rest of the points in the circuit are in $\mathcal{B}_{0}$. Let $\left(c^{0} C_{+} \cup\{-O\}, c^{0} C_{-}\right)$or $\left(c^{0} C_{+}, c^{0} C_{-} \cup\{-O\}\right)$ be that circuit. Similar arguments as in the star of $O$ prove that $\left(C_{+}, C_{-}\right)$is the copy in $\mathcal{B}_{0}$ of one of the nine circuits of $\mathcal{A}$ which supports a flip of $T$ and that $-O$ cannot be in the positive part of the circuit. Thus, the circuit is of the form $\left(c^{0} C_{+}, c^{0} C_{-} \cup\{-O\}\right)$, for one of the nine flippable circuits $\left(C_{+}, C_{-}\right)$of $T$.

We conclude that all the flippable circuits are of the form $\left(c^{0} C_{+}, c^{0} C_{-} \cup\{-O\}\right)$ or $\left(c^{n} C_{+}, c^{n} C_{-} \cup\{O\}\right)$, where $\left(C_{+}, C_{-}\right)$is a flippable circuit of $T$. This, in principle, gives 18 possibilities. However, for each circuit $\left(C_{+}, C_{-}\right)$of $T,\left(c^{0} C_{+}, c^{0} C_{-} \cup\{O\}\right)$ is a circuit of $\mathcal{A}_{n}$ if and only if $\left(c^{n} C_{+}, c^{n} C_{-} \cup\{O\}\right)$ is a circuit as well (this follows easily from the construction of the layers). The latter is a circuit if and only if $\left(c^{n} C_{+} \cup\{-O\}, c^{n} C_{-}\right)$is a circuit (since $O$ and $-O$ are opposite vectors). Finally, only one of $\left(c^{n} C_{+} \cup\{-O\}, c^{n} C_{-}\right)$ and $\left(c^{n} C_{+}, c^{n} C_{-} \cup\{-O\}\right)$ can be a circuit. This means that each flippable circuit of $T$ produces (at most) one instead of two flippable circuits in $T_{n}^{\prime}$. Although we do not prove it, it is easy to show that each flippable circuit of $T$ indeed produces a flippable circuit of $T_{n}^{\prime}$, or otherwise the link of $O$ could not be generic.

Remark 13. One may ask how many flips can be obtained in an acyclic version of the last construction. This amounts to removing the star of $-O$ and completing to a triangulation, perhaps with additional vertices, in Theorem 12. This case is more difficult to analyze because the simplices to be added in order to complete to a trian- 
gulation depend on the values chosen for the parameters $\alpha$ which perturb the points. The following argument shows that a number of flips lower or equal to 21 can be obtained. We think that a value of nine flips can be obtained with a more careful analysis.

Consider the point configuration $\mathcal{A}$ in $\mathbb{R}^{4}$ consisting of the origin $O$ and the following 15 points:

$$
\begin{aligned}
& t_{i j}=\frac{1}{\sqrt{2}}\left(\cos \left(\frac{2 \pi i}{3}-\beta\right), \sin \left(\frac{2 \pi i}{3}-\beta\right), \cos \left(\frac{2 \pi j}{3}-\beta\right), \sin \left(\frac{2 \pi j}{3}-\beta\right)\right), \\
& h_{i}=\left(\cos \left(\frac{2 \pi i}{3}\right), \sin \left(\frac{2 \pi i}{3}\right), 0,0\right), \quad v_{j}=\left(0,0, \cos \left(\frac{2 \pi j}{3}\right), \sin \left(\frac{2 \pi j}{3}\right)\right) .
\end{aligned}
$$

These are the same points of Section 4 except for the parameter $\beta$ in the points $t_{i j}$, which we take to be positive and smaller than $\pi / 12$. In these conditions the following 18 tetrahedra and 18 square pyramids are the facets of $\operatorname{conv}(\mathcal{A})$. For each $i=0,1,2$ and $j=0,1,2$ :

$$
\begin{aligned}
& {\left[h_{i}, t_{i, j}, t_{i, j+1}, t_{i+1, j}, t_{i+1, j+1}\right], \quad\left[h_{i}, h_{i+1}, t_{i+1, j}, t_{i+1, j+1}\right],} \\
& {\left[v_{j}, t_{i, j}, t_{i, j+1}, t_{i+1, j}, t_{i+1, j+1}\right], \quad\left[v_{j}, v_{j+1}, t_{i, j+1}, t_{i+1, j+1}\right] .}
\end{aligned}
$$

In other words, each of the 18 subprisms mentioned in Section 4 is broken into a square pyramid and a tetrahedron by the introduction of the parameter $\beta$. The only minimal nonsimplicial faces of $\operatorname{conv}(\mathcal{A})$ are the nine quadrilaterals $\left[t_{i, j}, t_{i+1, j}, t_{i, j+1}, t_{i+1, j+1}\right]$. The triangulation $T$ of Section 4 refines $\operatorname{conv}(\mathcal{A})$ and, thus, joining $T$ to $O$ we obtain a triangulation of $\mathcal{A}$, that we still call $T$.

The link of $O$ does not have the genericity property required in Theorem 8 . We can get this by changing the three points $t_{i i}$ to $\alpha O+(1-\alpha) t_{i i}$, for a small positive constant $\alpha$ (we are moving these three points slightly toward $O$ ). This perturbation breaks the coplanarity of the former nine quadrilaterals but makes the union of the simplices that we have so far nonconvex. The nonconvex parts can be filled in with the following six 4-simplices. For each $i=0,1,2$ :

$$
\left[h_{i}, t_{i, i}, t_{i, i+1}, t_{i+1, i}, t_{i+1, i+1}\right], \quad\left[v_{i}, t_{i, i}, t_{i, i+1}, t_{i+1, i}, t_{i+1, i+1}\right] .
$$

Now we can count the number of flips in this triangulation with the same arguments as in the proof of Theorem 12. The six quadrilaterals that have been perturbed convex produce six flips in the star of the central point $O$, and they are the only flips containing $O$ in a flippable facet. Part (ii) of Theorem 8 tells us that the other possible flips will have a flippable facet in one of the six extra 4-simplices added to the triangulation. Each of those simplices has three interior facets: the complements of $h_{i}$ (or $v_{i}$ ), $t_{i+1, i}$, and $t_{i, i+1}$ and one of them is common to two 4-simplices. This gives a total of 15 possible flippable facets and, together with the six flips at $O$, a total of 21 possible flips. 


\section{References}

1. M. Azaola and F. Santos, The graph of triangulations of $d+4$ points is 3-connected, manuscript, 1998.

2. L. Billera, P. Filliman, and B. Sturmfels, Constructions and complexity of secondary polytopes, $A d v$. in Math. 83 (1990), 155-179.

3. L. Billera, I.M. Gel'fand, and B. Sturmfels, Duality and minors of secondary polyhedra, J. Combin. Theory Ser. B 57 (1993), 258-268.

4. L. Billera, M. Kapranov, and B. Sturmfels, Cellular strings on polytopes, Proc. Amer. Math. Soc. 122(2) (1994), 549-555.

5. A. Björner, M. Las Vergnas, B. Sturmfels, N. White, and G. Ziegler, Oriented Matroids, Cambridge University Press, Cambridge, 1992.

6. J.A. de Loera, Triangulations of Polytopes and Computational Algebra, Ph.D. thesis, Cornell University, 1995.

7. J.A. de Loera, S. Hoşten, F. Santos, and B. Sturmfels, The polytope of all triangulations of a point configuration, Doc. Math. J. DMV 1 (1996), 103-119.

8. J.A. de Loera, F. Santos, and J. Urrutia, The number of geometric bistellar neighbors of a triangulation, Discrete Comput. Geom. 21(1) (1999), 131-142.

9. I.M. Gel'fand, M.M. Kapranov, and A.V. Zelevinsky, Multidimensional Determinants, Discriminants and Resultants, Birkhäuser, Boston, 1994.

10. L. Glaser, Geometrical Combinatorial Topology, Van Nostrand Reinhold, New York, 1970.

11. C.L Lawson, Software for $C^{1}$-interpolation, in Mathematical Software III (John Rice, ed.), pp. 161-194, Academic Press, New York, 1977.

12. C.W. Lee, Regular triangulations of convex polytopes, in Applied Geometry and Discrete MathematicsThe Victor Klee Festschrift (P. Gritzmann and B. Sturmfels, eds.), pp. 443-456, DIMACS series in Discrete Mathematics and Theoretical Computer Science, Vol. 4, AMS, Providence, RI, 1991.

13. R. MacPherson, Combinatorial differential manifolds, in Topological Methods in Modern Mathematics: a Symposium in Honor of John Milnor's Sixtieth Birthday, pp. 203-221, Publish or Perish, Houston, TX, 1993.

14. J. Rambau, Polyhedral subdivisions and projections of polytopes, Ph.D. Thesis, Fachbereich Mathematik, TU-Berlin, Shaker-Verlag, Aachen, 1996.

15. J. Rambau, Triangulations of cyclic polytopes and higher Bruhat orders, Mathematika 44 (1997), 162-194.

16. J. Rambau and F. Santos, The generalized Baues problem for cyclic polytopes, I, in "Combinatorics of convex polytopes" (K. Fukuda and G. H. Ziegler, eds.), European J. Combin., to appear.

17. J. Rambau and G.M. Ziegler, Projections of polytopes and the generalized Baues conjecture, Discrete Comput. Geom. 16(3) (1996), 215-237.

18. V. Reiner, The generalized Baues problem, in New Perspectives in Algebraic Combinatorics, HSRI Book Series, Cambridge University Press, to appear.

19. J. Richter-Gebert, Oriented matroids with few mutations, Discrete Comput. Geom. 10 (1993), 251-269.

20. F. Santos, Triangulations of oriented matroids, preprint, 1997, 77 pages. http://matsun1.unican.es/ $\sim$ santos/Articulos/OMtri.ps.gz

21. G. Ziegler, Lectures on Polytopes, Springer-Verlag, New York, 1994.

Received June 4, 1998, and in revised form October 10, 1998. 